\title{
Generalization of the analytical solution of neutron point kinetics equations with time-dependent external source
}

\author{
M. Seidi $\cdot$ S. Behnia $\cdot$ R. Khodabakhsh
}

Received: 23 July 2014/ Accepted: 5 September 2014/Published online: 23 September 2014

(C) The Author(s) 2014. This article is published with open access at Springerlink.com

\begin{abstract}
Point reactor kinetics equations with one group of delayed neutrons in the presence of the time-dependent external neutron source are solved analytically during the start-up of a nuclear reactor. Our model incorporates the random nature of the source and linear reactivity variation. We establish a general relationship between the expectation values of source intensity and the expectation values of neutron density of the sub-critical reactor by ignoring the term of the second derivative for neutron density in neutron point kinetics equations. The results of the analytical solution are in good agreement with the results obtained with numerical solution.
\end{abstract}

Keywords Neutron source $\cdot$ Delayed neutron · Point reactor kinetics $\cdot$ Start-up

\section{Introduction}

The neutron point kinetics (NPK) equations are a system of coupled nonlinear and stiff equations [1-3]. This system describes the nuclear parameters such as neutron density, reactivity and the precursor concentrations of delayed neutrons [4]. Calculations of these parameters are concerned with the reactor dynamics. The neutron density is one of the most important parameters in reactor dynamics [5-7]. According to the importance of neutron density in

\footnotetext{
M. Seidi · R. Khodabakhsh

Department of Physics, University of Urmia, Urmia, Iran

e-mail: r.khodabakhsh@urmia.ac.ir

S. Behnia $(\bowtie)$

Department of Physics, Urmia University of Technology,

Urmia, Iran

e-mail: s.behnia@sci.uut.ac.ir
}

the cold start-up stage, the external neutron source makes a significant contribution to the reactor power [8]. In most reactors, the reactivity is introduced mainly by discontinuous transferring of control rods which is, in practice, a linear function. Applying too much reactivity will cause a high increase in reactor power and can result in an overpressure accident in the reactor core [9]. Therefore, one should carefully study the influence of external neutron source on the reactor power $[10,11]$. So far, appropriate mathematical models have already been developed to study the sub-critical kinetics, such as including the constant external neutron source [6], approximation models [12-14] and prompt jump approximation (PJA) [9, 15, 16].

In this work we extend the analytical method to determine the effect of external source on the behavior of the neutron density during the start-up of a nuclear power reactor. The source of neutron is considered as a function of time.

This paper is organized as follows. In Sect. "Start-up process", a brief description of the start-up process is presented. In Sect. "External neutron source contribution", the mechanism of interaction between the control rod and external source is reviewed, and finally the authors discuss and interpret the results. The section is also followed by two appendices.

\section{Start-up process}

The start-up process of a reactor deals with the variation of reactivity in the system which occurs by raising the control rods in a discontinuous way [12]. So, the rule of neutron density variation is significant because it not only describes the relationship between lifting speed of the control rod, duration and speed of neutron density response, but also is 
helpful to the operator to control the reactor start-up from any accident $[9,17,18]$. The authors modified the analytical expressions for the calculation of neutron density from a set of physical and mathematical approximations [9, 12, 15]. Since the reactor is in sub-critical state in the cold start-up stage, the external neutron source cannot be ignored. Due to the low average temperature in the reactor core, here, the effect of temperature feedback can be ignored [9, 19]; consequently, the NPK equations with the external neutron source term for one group of delayed neutrons can be written as follows [8, 11, 12, 16]:

$$
\begin{gathered}
\frac{\mathrm{dn}(\mathrm{t})}{\mathrm{dt}}=\frac{\rho(t)-\beta}{l} n(t)+\lambda c(t)+q(t) \\
\frac{\mathrm{dc}(\mathrm{t})}{\mathrm{dt}}=\frac{\beta}{l} n(t)-\lambda c(t)
\end{gathered}
$$

where $n$ (neutron $\left.\mathrm{cm}^{-3}\right)$ is the neutron density, $c$ (neutron $\left.\mathrm{cm}^{-3}\right)$ is the precursor concentrations, $\beta$ is the total fraction of delayed neutrons, $\lambda\left(\mathrm{s}^{-1}\right)$ is the decay constant of delayed neutrons, $l(\mathrm{~s})$ is the prompt neutron generation time, $\rho$ is the reactivity as a function of time, and $q$ (neutron $\left.\mathrm{cm}^{-3} \mathrm{~s}^{-1}\right)$ is the external neutron source.

To have a general solution, the variation of external neutron source with respect to time is considered. Let us first consider the external neutron source defined as the ratios of polynomials of degree $N$ :

$q(t)=\sum_{n=0}^{N} q_{n} t^{n}$

We denote their reactivity by:

$\rho(t)= \begin{cases}\rho_{0}+r t & 0 \leq t<t_{0} \\ \rho_{0}+r t_{0} & t \geq t_{0}\end{cases}$

where $\rho_{0}$ is the initial subcritical reactivity, $r\left(\mathrm{~s}^{-1}\right)$ represents the ramp reactivity addition rates and $t_{0}(\mathrm{~s})$ is the time for taking out the rods.

Eliminating the dependency of precursor concentration, the differential equation that governs the neutron density during the withdrawal of the control rods is given by [9, 12]:

$$
\begin{aligned}
l \frac{\mathrm{d}^{2} \mathrm{n}(\mathrm{t})}{\mathrm{dt}^{2}}= & (\rho-\beta-\lambda l) \frac{\mathrm{dn}(\mathrm{t})}{\mathrm{dt}}+\left(\lambda \rho+\frac{\mathrm{d} \rho(\mathrm{t})}{\mathrm{dt}}\right) n(t) \\
& +l\left(\lambda q(t)+\frac{\mathrm{dq}(\mathrm{t})}{\mathrm{dt}}\right)
\end{aligned}
$$

Since $l \frac{\mathrm{d}^{2} \mathrm{n}(\mathrm{t})}{\mathrm{dt}^{2}}$ is much smaller than the other terms in Eq. (3), we "Ignore the second term derivative" (ISD). So, the only approximation which we used in the calculations is ISD [9, 12]. According to the ISD, one can write: $(\rho-\beta-\lambda l) \frac{\mathrm{dn}(\mathrm{t})}{\mathrm{dt}}+\left(\lambda \rho+\frac{\mathrm{d} \rho(\mathrm{t})}{\mathrm{dt}}\right) n(t)=-l\left(\lambda q(t)+\frac{\mathrm{dq}(\mathrm{t})}{\mathrm{dt}}\right)$.

According to the data of some thermal reactors, i.e. $\lambda=0.001 \mathrm{~s}^{-1}, \beta=0.0075, \rho_{0}=-0.006,1=0.0015 \mathrm{~s}$, the assumption of $\beta-\rho \gg \lambda l$ for very small ramp rate reactivities is valid [12]. To find the analytical solution, provided $n=n_{1}, \rho_{1}=\rho_{0}+r t, k_{1}=\frac{\lambda \rho_{0}+r}{r}$ and $k_{2}=\frac{\beta-\rho_{0}}{r}$, the equivalent form of Eq. (4) is rearranged as follows:

$$
\frac{\mathrm{dn}_{1}}{\mathrm{dt}}+\left(\frac{k_{1}+\lambda t}{t-k_{2}}\right) n_{1}=-\frac{l}{r\left(t-k_{2}\right)} \sum_{n=0}^{N}\left(\lambda q_{n}+(n+1) q_{n+1}\right) t^{n} .
$$

Considering $\left(u=t-k_{2}, \eta=k_{1}+\lambda k_{2}-1\right)$ [12], Eq. (5) reduces to:

$$
\begin{aligned}
n_{1}(t)= & \frac{e^{-\lambda u}}{u^{\eta+1}}\left(-\frac{l}{r} \int \mathrm{dt} \mathrm{u}^{\eta} \mathrm{e}^{\lambda \mathrm{u}} \sum_{\mathrm{n}=0}^{\mathrm{N}} \sum_{\mathrm{m}=0}^{\mathrm{n}}\left(\begin{array}{l}
\mathrm{n} \\
\mathrm{m}
\end{array}\right) \mathrm{k}_{2}^{\mathrm{m}}\left(\lambda \mathrm{q}_{\mathrm{n}}\right.\right. \\
& \left.\left.+(n+1) q_{n+1}\right) u^{n-m}+A\right) .
\end{aligned}
$$

One can write (for more details see "Appendix A"):

$n_{1}(t)=\frac{e^{-\lambda u}}{u^{\eta+1}}\left(\sum_{n=0}^{N} \sum_{m=0}^{n} a_{n m} \gamma(\eta+n-m+1,-\lambda u)+A\right)$

$a_{n m}=\left(\left(-\frac{l}{r}\right)\left(-\frac{1}{\lambda}\right)^{n+\eta-m+1} k_{2}^{m}\left(\lambda q_{n}+(n+1) q_{n+1}\right)\right)\left(\begin{array}{l}n \\ m\end{array}\right)$.

It is possible to determine the integration constant $(A)$ by using initial condition, $\left(n_{1}(0)=n_{0}\right)$, and so we have:

$A=\left(-\sum_{n=0}^{N} \sum_{m=0}^{n} a_{n m} \gamma(\eta+n-m+1,-\lambda u)+\frac{n_{0}\left(-k_{2}\right)^{\eta+1}}{e^{\lambda k_{2}}}\right)$.

For $t=t_{0}$, the reactivity will reach a constant value $\left(\rho_{2}=\rho_{0}+r t_{0}\right)$. At this time, neutron density is equal to $n_{2}(t)=n_{2}$. Therefore, in this stage of introducing reactivity, the differential equation is given by:

$\frac{\mathrm{dn}_{2}}{\mathrm{dt}}+s n_{2}=\sum_{n=0}^{N} b_{n} t^{n}$

where $s=\frac{\lambda\left(\rho_{0}+r t_{0}\right)}{\rho_{0}+r t_{0}-\beta}$ and $b_{n}=-\frac{l\left(\lambda q_{n}+(n+1) q_{n+1}\right)}{\rho_{0}+r t_{0}-\beta}$. Finally, the solution of Eq. (8) can be obtained from the integrating factor method (for more details see "Appendix B"): 
$n_{2}(t)=\left(e^{-s t}\left(\sum_{n=0}^{N} b_{n}\left(-\frac{1}{s}\right)^{n+1} \Gamma(n+1,-s t)+B\right)\right)$.

The integral constant can be calculated by applying the continuity condition $\left(n_{2}\left(t_{0}\right)=n_{1}\left(t_{0}\right)\right)$ :

$$
\begin{aligned}
B= & \frac{e^{s t_{0}-\lambda\left(t_{0}-k_{2}\right)}}{\left(t_{0}-k_{2}\right)^{\eta+1}}\left(\sum _ { n = 0 } ^ { N } \sum _ { m = 0 } ^ { n } a _ { n m } \left[\gamma\left(\eta+n-m+1,-\lambda\left(t_{0}-k_{2}\right)\right)\right.\right. \\
& \left.-\gamma\left(\eta+n-m+1, \lambda k_{2}\right)\right]-\sum_{n=0}^{N} b_{n}\left(-\frac{1}{s}\right)^{n+1} \\
& \left.\times \Gamma\left(n+1,-s t_{0}\right)+\frac{n_{0}\left(-k_{2}\right)^{\eta+1}}{e^{\lambda k_{2}}}\right)
\end{aligned}
$$

In summary, utilizing Eqs. (6) and (9), the neutron density was calculated when the core of the reactor was interpolated by an external source at the start-up stage. In the following, the neutron density was calculated for the case of a sinusoidal source, which is very close to a real system. In addition, the constant source was also considered to examine the validity of the obtained results.

\section{External neutron source contribution}

The analytical solution is calculated with two terms: one as a function of sinusoidal fluctuation in the external source, and the other as a function of constant source. Using the sinusoidal solution, the importance of the source term in the reactor kinetics analysis is better manifest.

\section{Constant external neutron source}

By calculating the neutron density for constant source, $\left(q=q_{0}\right)$, we have tried to show that the obtained result reduces to Palma et al.'s result [12]. From density equation definition of Eq. (6), we can write:

$$
n_{1}(t)=\left(\frac{e^{-\lambda u}}{u^{\eta+1}}\left(a_{00} \gamma(\eta+1,-\lambda u)+A\right)\right)
$$

where constants $a_{00}$ and $A$ are defined by:

$a_{00}=\frac{l q_{0}}{r(-\lambda)^{\eta}}, \quad A=\left(\frac{-l q_{0}}{r(-\lambda)^{\eta}} \gamma\left(\eta+1, \lambda k_{2}\right)+\frac{n_{0}\left(-k_{2}\right)^{\eta+1}}{e^{\lambda k_{2}}}\right)$.

The complete solution of Eq. (8) with external constant source is given by:

$$
n_{2}(t)=\left(e^{-s t}\left(\left(-\frac{b_{0}}{s}\right) \Gamma(1,-s t)+B\right)\right)
$$

In deriving $n_{2}(t)$, we used the continuity condition. In Eq. (12), $B$ is the integration constant given in terms of the incomplete gamma function as:

$$
\begin{aligned}
B= & \left(\frac { e ^ { s t _ { 0 } - \lambda ( t _ { 0 } - k _ { 2 } ) } } { ( t _ { 0 } - k _ { 2 } ) ^ { \eta + 1 } } \left(a_{00} \gamma\left(\eta+1, \lambda\left(k_{2}-t_{0}\right)\right)\right.\right. \\
& \left.+A)+\left(\frac{b_{0}}{s}\right) \Gamma\left(1, s t_{0}\right)\right) .
\end{aligned}
$$

Therefore, Eqs. (11) and (12) are in agreement with previous results [Eqs. (16), (24) [12]]. These solutions are expressed in terms of incomplete gamma function and related to the probability integral of the chi-squared distribution, as discussed and tabulated by Abramowitz and Stegun [20].

\section{Sinusoidal fluctuation}

Due to fluctuation of neutron source around a constant value, the source is time dependent. Accounting for the sinusoidal external neutron source, $q(t)=q_{0} \sin \omega t\left(\omega=\frac{2 \pi}{T}\right.$,

\begin{tabular}{|c|c|c|c|c|c|}
\hline \multirow{2}{*}{$\begin{array}{l}\text { Time } \\
t(\mathrm{~s})\end{array}$} & \multirow{2}{*}{$\begin{array}{l}\text { Numerical solution without ISD }\left(n_{1}\right) \\
n_{1}(t) \times 10^{8}\end{array}$} & \multicolumn{2}{|c|}{$\begin{array}{l}\text { Numerical solution with } \\
\text { ISD }\left(n_{2}\right)\end{array}$} & \multicolumn{2}{|c|}{$\begin{array}{l}\text { Analytical solution with } \\
\text { ISD }\left(n_{3}\right)\end{array}$} \\
\hline & & $n_{2}(t) \times 10^{8}$ & $(\text { RPES })_{12}$ & $n_{3}(t) \times 10^{8}$ & $(\text { RPEs })_{13}$ \\
\hline 2 & 2.53546 & 2.53761 & 0.04 & 2.53470 & 0.03 \\
\hline 4 & 2.57417 & 2.57642 & 0.09 & 2.57051 & 0.14 \\
\hline 6 & 2.61411 & 2.61648 & 0.91 & 2.60748 & 0.25 \\
\hline 8 & 2.65536 & 2.65784 & 0.93 & 2.64566 & 0.36 \\
\hline 10 & 2.69797 & 2.70058 & 0.97 & 2.68513 & 0.47 \\
\hline 20 & 2.70175 & 2.70178 & 0.01 & 2.68638 & 0.57 \\
\hline 30 & 2.70291 & 2.70436 & 0.05 & 2.68763 & 0.56 \\
\hline 40 & 2.70415 & 2.70542 & 0.05 & 2.68881 & 0.57 \\
\hline 50 & 2.70526 & 2.70649 & 0.04 & 2.69051 & 0.54 \\
\hline
\end{tabular}
where $\omega\left(\mathrm{s}^{-1}\right)$ and $T(\mathrm{~s})$ are angular frequency and period of
Table 1 The neutron densities and the relative errors for twostage insertion of reactivity in the presence of a constant external neutron source 


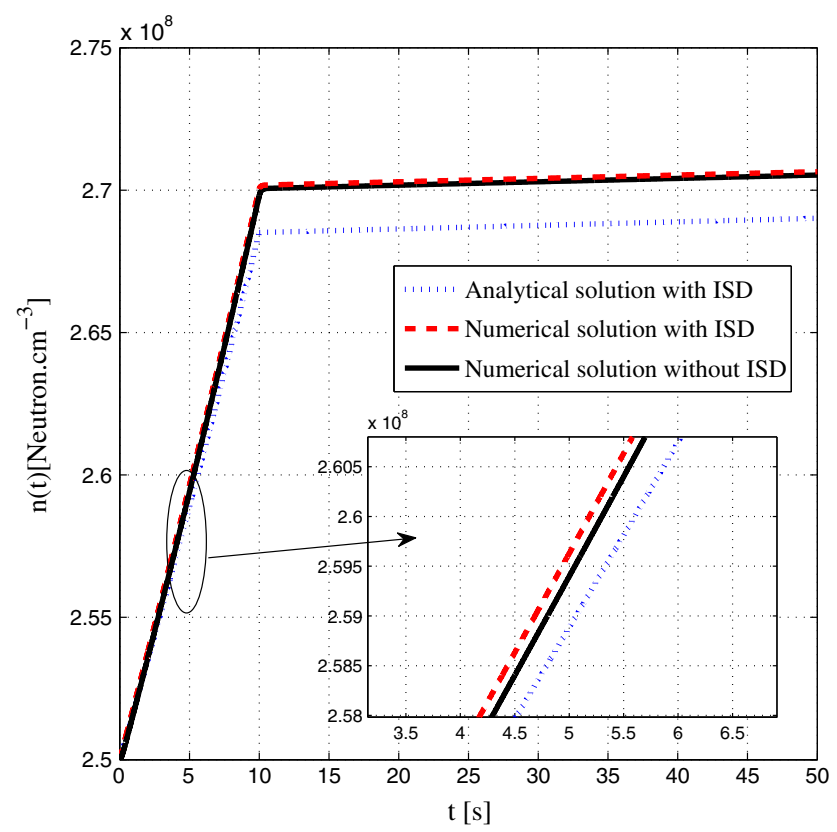

Fig. 1 Comparison of the analytical solution with the numerical solutions for $r=0.0001 \mathrm{~s}^{-1}, \rho_{0}=-0.006, q=10^{9} \frac{\text { neutron }}{\mathrm{cm}^{3} \mathrm{~s}}$ and $t_{0}=$ $10 \mathrm{~s}$ in the presence of a constant external neutron source

source, respectively) can be useful for indicating the environment effects in neutron density [16]. Let us recall Eq. (6) for neutron density coefficients:

$a_{n m}=\left(-\frac{1}{\lambda}\right)^{\eta+2 n-m+1}\left(\frac{l q_{0}}{r}\right)\left(\frac{(-1)^{n} k_{2}^{m} \omega^{2 n+1}}{m !(2 n-m+1) !}\right)_{2 n+1 \geq m}$ $b_{n m}=\left(-\frac{1}{\lambda}\right)^{\eta+2 n-m+1}\left(-\frac{l q_{0}}{r}\right)\left(\frac{(-1)^{n} k_{2}^{m} \omega^{2 n+1}}{m !(2 n-m) !}\right)_{2 n \geq m}$

Neutron density can be presented as:

$$
\begin{aligned}
n_{1}(t)= & \frac{e^{-\lambda u}}{u^{\eta+1}}\left(\sum_{n=0}^{N} \sum_{m=0}^{2 n+1} a_{\mathrm{rmnm}} \gamma(\eta+2 n-m+2,-\lambda u)\right. \\
& \left.+\sum_{n=0}^{N} \sum_{m=0}^{2 n} b n m \gamma(\eta+2 n-m+1,-\lambda u)+A\right) .
\end{aligned}
$$

After imposing the initial condition, $n_{1}(0)=n_{0}$, one can determine the constant $A$ from Eq. (15). So, we have:

$$
\begin{aligned}
A=\frac{n_{0}\left(-k_{2}\right)^{\eta+1}}{e^{\lambda k_{2}}} & -\left(\sum_{n=0}^{N} \sum_{m=0}^{2 n+1} \operatorname{anm} \gamma\left(\eta+2 n-m+2, \lambda k_{2}\right)\right. \\
& \left.+\sum_{n=0}^{N} \sum_{m=0}^{2 n} b n m \gamma\left(\eta+2 n-m+1, \lambda k_{2}\right)\right) .
\end{aligned}
$$

The solution of Eq. (8), in the second stage from insertion of reactivity, can be calculated from the integration factor method:

$n_{2}(t)=e^{-s t}\left(\sum_{n=0}^{N}\left(c_{n} \Gamma(2 n+2,-s t)+d_{n} \Gamma(2 n+1,-s t)\right)+B\right)$

where
Fig. 2 Multiplication factor as a function of time and initial reactivity for very small ramp rate reactivity $\left(r=0.00001 \mathrm{~s}^{-1}\right)$

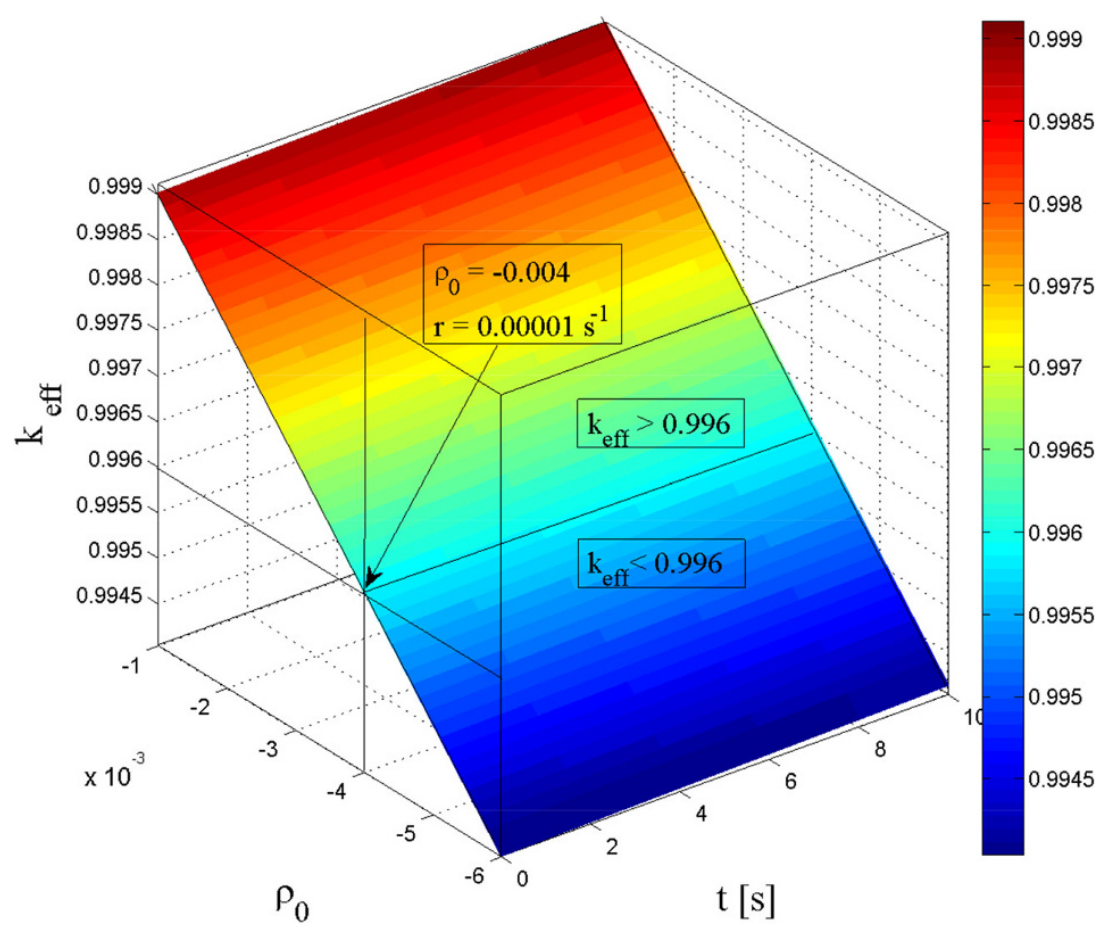




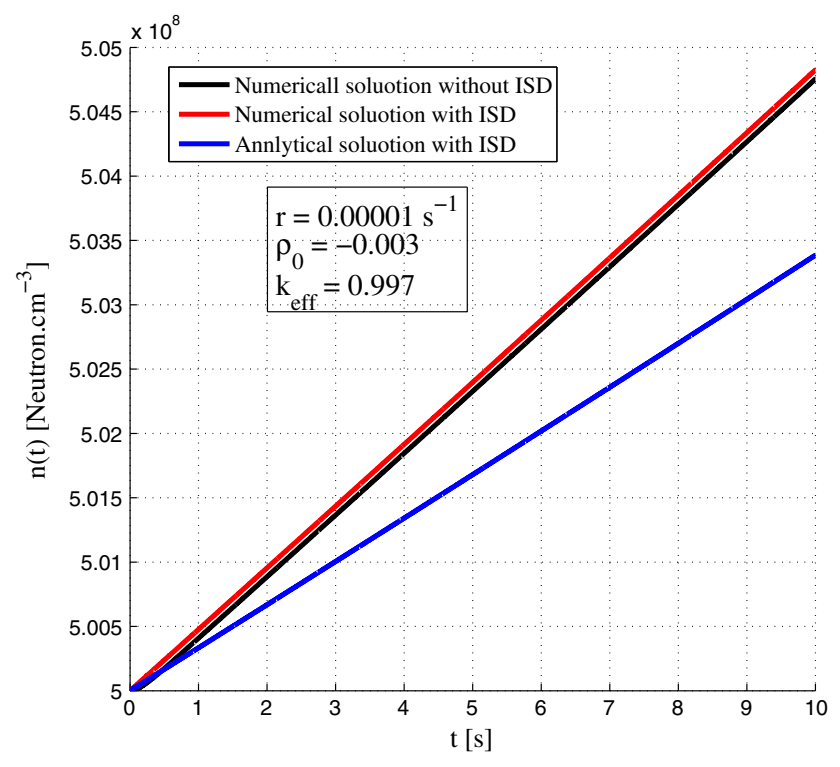

Fig. 3 Multiplication factor as a function of time and initial reactivity for very small ramp rate reactivity $\left(r=0.00001 \mathrm{~s}^{-1}\right)$

$c_{n}=\left(-\frac{l q_{0} \lambda}{\rho_{0}+r t_{0}-\beta}\right)\left(\frac{(-1)^{n} \omega^{2 n+1}}{(2 n+1) !}\right)\left(\frac{1}{s}\right)^{2 n+2}$

$d_{n}=\left(\frac{l q_{0} \lambda}{\rho_{0}+r t_{0}-\beta}\right)\left(\frac{(-1)^{n} \omega^{2 n+2}}{(2 n) !}\right)\left(\frac{1}{s}\right)^{2 n+1}$.

In Eq. (16), $B$ is the integration constant given in terms of the incomplete gamma functions, which can be obtained from continuity condition $\left(n_{1}\left(t_{0}\right)=n_{2}\left(t_{0}\right)\right)$ :

$$
\begin{aligned}
B= & \frac{e^{s t_{0}-\lambda\left(t_{0}-k_{2}\right)}}{\left(t_{0}-k_{2}\right)^{\eta+1}}\left(\sum_{n=0}^{N} \sum_{m=0}^{2 n+1} a_{\mathrm{nm}} \gamma\left(\eta+2 n-m+2, \lambda\left(k_{2}-t_{0}\right)\right)\right. \\
& \left.+\sum_{n=0}^{N} \sum_{m=0}^{2 n} b_{\mathrm{nm}} \gamma\left(\eta+2 n-m+1, \lambda\left(k_{2}-t_{0}\right)\right)+A\right) \\
& -\left(c_{n} \Gamma\left(2 n+2,-s t_{0}\right)+d_{n} \Gamma\left(2 n+1,-s t_{0}\right)\right) .
\end{aligned}
$$

Equations (15) and (16) represent the complete solutions of Eqs. (15) and (8) in the presence of the sinusoidal external neutron source.

\section{Results and discussions}

Neutron external source play an important role during the start-up of a nuclear reactor. Therefore, the analytical solutions of neutron point kinetics equations in the presence of an external source are important in predicting the variation of neutron population during the start-up of a nuclear reactor. Here, the analyses are presented for the thermal reactor with the following parameters [9, 12]: $\lambda=0.001 \mathrm{~s}^{-1}, \quad l=0.0015 \mathrm{~s}, \quad \beta=0.0075, \quad r=0.0001 \mathrm{~s}^{-1}$, $\rho_{0}=-0.006$, and $t_{0}=10 \mathrm{~s}$. Equations (6) and (9) are the solutions of the neutron point kinetics equations with onegroup delayed neutron during the start-up of the thermal reactor in a two-stage insertion of reactivity. They are valid for any function that can be expressed by a power series. Due to fluctuation of the neutron source around a mean value, the source is actually time dependent. The general solutions of NPK equations can well describe neutron density response to any external neutron source, both quantitatively and qualitatively. To verify the validity of the analytical solutions, the variation of neutron density with constant and sinusoidal external neutron source was calculated. Specially, for $n=0$ and $m=0$, the neutron density was obtained in the presence of a constant source [see Eqs. (11), (12)]. This result is in line with Palma et al.'s work [12]. In this work, numerical calculations were carried out with generalized Runge-Kutta (GRK) method. The relative percentage errors (RPEs) of the neutron density using analytical and numerical solutions are defined as follows:

$$
\begin{aligned}
& (\text { RPEs })_{12}=\left|\frac{n_{1}(t)-n_{2}(t)}{n_{1}(t)}\right| \times 100 \\
& (\text { RPEs })_{13}=\left|\frac{n_{1}(t)-n_{3}(t)}{n_{1}(t)}\right| \times 100,
\end{aligned}
$$

Table 2 The neutron densities and the relative errors for the

\begin{tabular}{|c|c|c|c|c|c|}
\hline \multirow{2}{*}{$\begin{array}{l}\text { Time } \\
\rho_{0}\end{array}$} & \multirow{2}{*}{$\begin{array}{l}\text { Numerical solution without ISD }\left(n_{1}\right) \\
n_{1}(t) \times 10^{8}\end{array}$} & \multicolumn{2}{|c|}{$\begin{array}{l}\text { Numerical solution with } \\
\text { ISD }\left(n_{2}\right)\end{array}$} & \multicolumn{2}{|c|}{$\begin{array}{l}\text { Analytical solution with } \\
\text { ISD }\left(n_{3}\right)\end{array}$} \\
\hline & & $n_{2}(t) \times 10^{8}$ & $(R P E S)_{12}$ & $n_{3}(t) \times 10^{8}$ & $(R P E s)_{13}$ \\
\hline-0.006 & 2.51850 & 2.51871 & 0.01 & 2.50754 & 0.43 \\
\hline-0.005 & 3.02397 & 3.02427 & 0.01 & 3.01219 & 0.39 \\
\hline-0.004 & 3.78256 & 3.78300 & 0.01 & 3.76987 & 0.33 \\
\hline-0.003 & 5.04754 & 5.04825 & 0.01 & 5.03385 & 0.27 \\
\hline-0.002 & 7.57880 & 7.58010 & 0.02 & 7.56416 & 0.19 \\
\hline-0.001 & 15.1761 & 15.1793 & 0.02 & 15.1615 & 0.10 \\
\hline
\end{tabular}
insertion of ramp reactivity in the presence of a constant external neutron source very close to criticality 


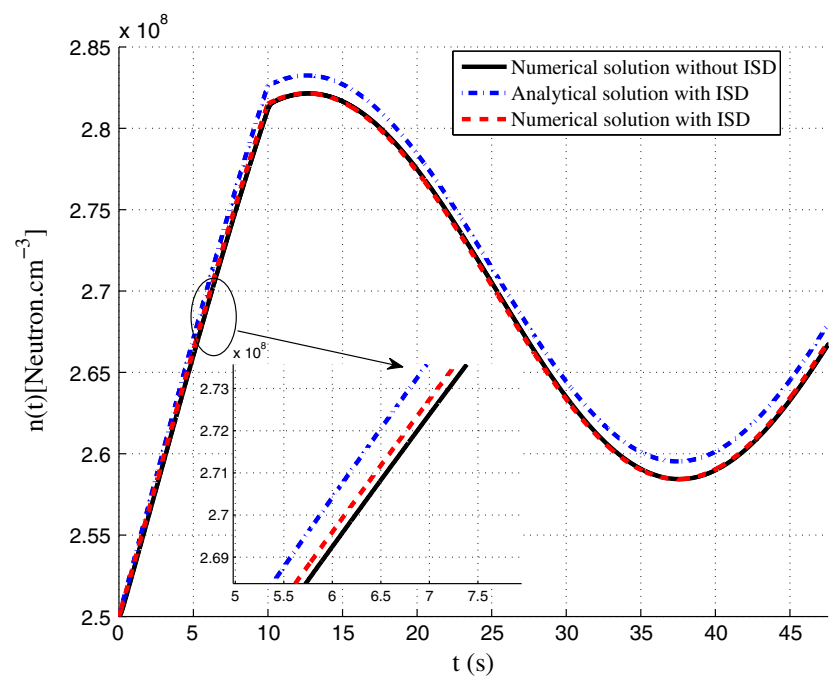

Fig. 4 Comparison of the analytical solution with the numerical solutions for $r=0.0001 \mathrm{~s}^{-1}, \rho_{0}=-0.006, q_{0}=10^{8} \frac{\text { neutron }}{\mathrm{cm}^{3} \mathrm{~s}}, T=50 \mathrm{~s}$ and $t_{0}=10 \mathrm{~s}$ in the presence of a sinusoidal external neutron source

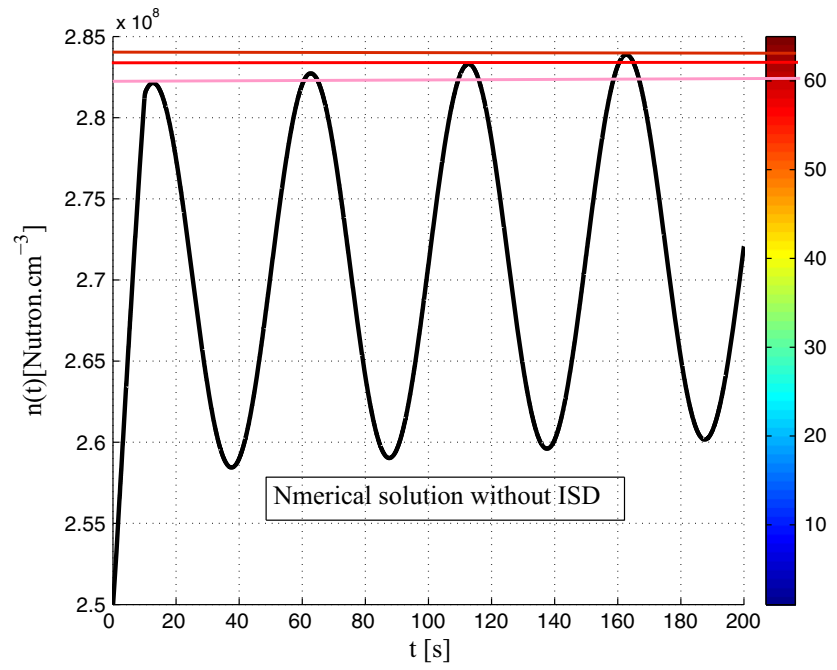

Fig. 5 The variation of neutron density during start-up for $r=0.0001 \mathrm{~s}^{-1}, \quad \rho_{0}=-0.006, \quad q_{0}=10^{8} \frac{\text { neutron }}{\mathrm{cm}^{3} \mathrm{~s}}, \quad T=50 \mathrm{~s} \quad$ and $0 \leq t \leq 200 \mathrm{~s}$ in the presence of a sinusoidal external neutron source, which shows an increase in the neutron density with time, as expected

wherethe neutron densities of $n_{1}, n_{2}$ and $n_{3}$ are the solutions of numerical calculations without ISD, with ISD and analytical calculation with ISD, respectively. According to Table 1, the RPEs are smaller than $1 \%$, and the resultant RPEs are acceptable for engineering applications. So, the results of the analytical calculations are in a good agreement with the numerical results (see Fig. 1). Studying neutron density with conditions close to the critical state and small ramp rate reactivities $(r \ll 1)$ is important for reactor control and safety. Effective multiplication factor $\left(k_{\text {eff }}\right)$ values, close to the critical state is shown in Fig. 2. As an example, Fig. 3 shows the numerical and analytical solution results for $r=10^{-5} \mathrm{~s}^{-1}, \rho_{0}=-0.003$ and $k_{\text {eff }}=0.9965$, which are in good agreement with each other. Data in Table 2 show that the RPEs of the numerical and analytical solution are less than $0.5 \%$ and also the RPEs decrease for $k_{\text {eff }} \longrightarrow 1$ and $r \ll 1$. So, the ISD approximation is valid for neutronic calculations very close to criticality and $r \ll 1$.

For $n=2 k+1$ and $k=0,1,2, \ldots$ the power series external source is:

$$
q(t)=\left(\sum_{n=0}^{N} a_{n} t^{n}\right) \equiv\left(\sum_{k=0}^{N} a_{2 k+1} t^{2 k+1}\right)=q_{0} \sin (\omega \mathrm{t}) .
$$

Such a phenomenon is also explained by Hetrick [16]. As a result, in the case of sinusoidal source with a small amplitude of oscillations, the constant source approximation is applicable at the start-up process [21, 22].

As an example, the neutron density using analytical solution for $n=20, m=0, . .9, T=50 \mathrm{~s}$ and $q_{0}=10^{8}$ (blue dashed-dotted line) was compared with numerical results, with ISD and without ISD in Fig. 4. After raising the control rods and during sturt-up, $10 \mathrm{~s} \leq \mathrm{t} \leq 200 \mathrm{~s}$, the neutron density increases with time, as shown in Fig. 5. Regarding Table 3, the RPEs do not reach $1 \%$. Therefore, the results of numerical and analytical methods are the same.

\section{Conclusion}

Since the external neutron source term is very important at the start-up stage, it cannot be ignored. Due to the random nature of the external neutron source, we can take a timedependent source. Therefore, in this work, an analytical solution is presented for any neutron external source that can be found in terms of a power series. Analytical solutions in a general form are as in Eqs. (6) and (9). In particular, analytical solutions are obtained for the constant [12] and sinusoidal sources [16]. Numerical and analytical results in both cases are in a good agreement with each other.

Open Access This article is distributed under the terms of the Creative Commons Attribution License which permits any use, distribution, and reproduction in any medium, provided the original author(s) and the source are credited.

\section{Appendix A}

Using transformation of variable $u=t-k_{2}$ and replacing in Eq. (5), we have: 
Table 3 The neutron densities and the relative errors for twostage insertion of reactivity in the presence of a sinusoidal external neutron source

\begin{tabular}{|c|c|c|c|c|c|}
\hline \multirow{2}{*}{$\begin{array}{l}\text { Time } \\
t(\mathrm{~s})\end{array}$} & \multirow{2}{*}{$\begin{array}{l}\text { Numerical solution without ISD }\left(n_{1}\right) \\
n_{1}(t) \times 10^{8}\end{array}$} & \multicolumn{2}{|c|}{$\begin{array}{l}\text { Numerical solution with } \\
\text { ISD }\left(n_{2}\right)\end{array}$} & \multicolumn{2}{|c|}{$\begin{array}{l}\text { Analytical solution with } \\
\text { ISD }\left(n_{3}\right)\end{array}$} \\
\hline & & $n_{2}(t) \times 10^{8}$ & $(R P E s)_{12}$ & $n_{3}(t) \times 10^{8}$ & $(R P E s)_{13}$ \\
\hline 2 & 2.56183 & 2.56537 & 0.14 & 2.56851 & 0.67 \\
\hline 4 & 2.62788 & 2.63130 & 0.13 & 2.63726 & 0.36 \\
\hline 6 & 2.69257 & 2.69612 & 0.13 & 2.70427 & 0.43 \\
\hline 8 & 2.75431 & 2.75779 & 0.13 & 2.76780 & 0.49 \\
\hline 10 & 2.81185 & 2.81538 & 0.12 & 2.82623 & 0.51 \\
\hline 20 & 2.77479 & 2.77338 & 0.05 & 2.78378 & 0.32 \\
\hline 30 & 2.63492 & 2.63345 & 0.06 & 2.64347 & 0.32 \\
\hline 40 & 2.58991 & 2.59042 & 0.02 & 2.60039 & 0.40 \\
\hline 50 & 2.70350 & 2.70533 & 0.08 & 2.71532 & 0.44 \\
\hline
\end{tabular}

$$
\begin{aligned}
\frac{\mathrm{dn}_{1}}{\mathrm{dt}} & +\left(\frac{\eta+1}{u}+\lambda\right) n_{1} \\
& =-\frac{l}{r u} \sum_{n=0}^{N}\left(\lambda q_{n}+(n+1) q_{n+1}\right)\left(u+k_{2}\right)^{n}
\end{aligned}
$$

where $\eta=k_{1}+\lambda k_{2}-1$. Also using binomial expansion as follows:

$$
\left(u+k_{2}\right)^{n}=\left(\sum_{m=0}^{n}\left(\begin{array}{l}
n \\
m
\end{array}\right) k_{2}^{m} u^{n-m}\right),
$$

one can write:

$$
\begin{aligned}
& \frac{\mathrm{dn}_{1}(\mathrm{t})}{\mathrm{dt}}+\left(\frac{\eta+1}{u}+\lambda\right) n_{1} \\
& =\left(-\frac{l}{r} \sum_{n=0}^{N} \sum_{m=0}^{n}\left(\begin{array}{l}
n \\
m
\end{array}\right) k_{2}^{m}\left(\lambda q_{n}+(n+1) q_{n+1}\right) u^{n-m-1}\right),
\end{aligned}
$$

where constant $d n m$ are defined as follows:

$d n m=\left(-\frac{l}{r}\left(\begin{array}{l}n \\ m\end{array}\right) k_{2}^{m}\left(\lambda q_{n}+(n+1) q_{n+1}\right)\right)$

$\frac{\mathrm{dn}_{1}(\mathrm{t})}{\mathrm{dt}}+\left(\frac{\eta+1}{u}+\lambda\right) n_{1}=\sum_{n=0}^{N} \sum_{m=0}^{n} d n m u^{n-m-1}$.

Equation (19) can be solved through the use of the integrating factor method. Therefore, we get:

$n_{1}(t)=\frac{e^{-\lambda u}}{u^{\eta+1}}\left(\sum_{n=0}^{N} \sum_{m=0}^{n} d n m \int d u e^{\lambda u} u^{\eta+n-m}+A\right)$.

Using the transformation of variable $y=-\lambda u$ and $\alpha=\eta+n-m+1$, we have:

$n_{1}(t)=\frac{e^{-\lambda u}}{u^{\eta+1}}\left(\sum_{n=0}^{N} \sum_{m=0}^{n} d_{n m}\left(-\frac{1}{\lambda}\right)^{\eta+n-m+1} \int_{0}^{t} d y e^{-y} y^{\alpha-1}+A\right)$ $a n m=\operatorname{dnm}\left(-\frac{1}{\lambda}\right)^{\eta+n-m+1}$

where

$\gamma(\alpha, t)=\int_{0}^{t} \mathrm{dye}^{-\mathrm{y}} \mathrm{y}^{\alpha-1}$

The solution of Eq. (21) can be written as:

$$
n_{1}(t)=\frac{e^{-\lambda u}}{u^{\eta+1}}\left(\sum_{n=0}^{N} \sum_{m=0}^{n} a_{n m} \gamma(\eta+n-m+1,-\lambda u)+A\right)
$$

\section{Appendix B}

Incomplete gamma function is defined as:

$\Gamma(\alpha, t)=\int_{t}^{\infty} \mathrm{dye}^{-\mathrm{y}} \mathrm{y}^{\alpha-1}$

Equation (9) can be solved through the use of the integrating factor method. Therefore we get:

$n_{2}(t)=e^{-s t}\left(\sum_{n=0}^{N} b_{n} \int \mathrm{dt} \mathrm{e}^{s \mathrm{~s}} \mathrm{t}^{\mathrm{n}}+\mathrm{B}\right)$.

Using $y=-s t$, one can write:

$n_{2}(t)=e^{-s t}\left(\sum_{n=0}^{N} b_{n}\left(-\frac{1}{s}\right)^{n+1} \int \mathrm{dy} \mathrm{e}^{-\mathrm{y}} \mathrm{y}^{\mathrm{n}}+\mathrm{B}\right)$

, so we have:

$n_{2}(t)=e^{-s t}\left(\sum_{n=0}^{N} b_{n}\left(-\frac{1}{s}\right)^{n+1} \Gamma(n+1,-s t)+B\right)$. 


\section{References}

1. Chen, W.Z., Hao, J., Chen, L., Li, H.: Solution of point reactor neutron kinetics equations with temperature feedback by singularly perturbed method. Sci. Technol. Nucl. Install., p 6 (2013) (Article ID 261327)

2. Li, H., Chen, W.Z., Luo, L., Zhu, Q.: A new integral method for solving the point reactor neutron kinetics equations. Ann. Nucl. Energy 36, 427-432 (2009)

3. Chen, W.Z., Guo, L., Zhu, B., Li, H.: Accuracy of analytical methods for obtaining supercritical transients with temperature feedback. Progr. Nucl. Energy 49, 290-302 (2007)

4. Nahla, A.A.: Taylors series method for solving the nonlinear point kinetics equations. Nucl. Eng. Design 241, 1592-1595 (2011)

5. Nahla, A.A.: Analytical solution to solve the point reactor kinetics equations. Nucl. Eng. Design 241, 1622-1629 (2010)

6. Aboanber, A.E., Nahla, A.A.: Adaptive matrix formation (AMF) method of spacetime multi group reactor kinetics equations in multidimensional model. Ann. Nucl. Energy 34, 113-119 (2007)

7. Sathiyasheela, T.: Power series solution method for solving point kinetics equations with lumped model temperature and feedback. Ann. Nucl. Energy 36, 246-251 (2009)

8. Sathiyasheela, T.: Inhomogeneous point kinetics equations and the source contribution. Nucl. Eng. Design 241, 4183-4191 (2010)

9. Zhang, F., Chen, W.Z., Gui, X.W.: Analytic method study of point-reactor kinetic equation when cold start-up. Ann. Nucl. Energy 35, 746-749 (2008)

10. Peinetti, F., Nicolino, C., Ravetto, P.: Kinetics of a point reactor in the presence of reactivity oscillations. Ann. Nucl. Energy 33, 1189-1195 (2006)

11. Polo-Labarrios, M.A., Espinosa-Paredes, G.: Application of the fractional neutron point kinetic equation: Start-up of a nuclear reactor. Ann. Nucl. Energy 46, 37-42 (2012)
12. Palma, A.D.P., Martinez, S.A., Gonalves, A.C.: Analytical solution of point kinetics equations for linear reactivity variation during the start-up of a nuclear reactor. Ann. Nucl. Energy 36, 1469-1471 (2009)

13. Hamieh, S.D., Saidinezhad, M.: Analytical solution of the point reactor kinetics equations with temperature feedback. Ann. Nucl. Energy 42, 148-152 (2012)

14. Li, H., Chen, W., Zhang, F., Chen, Z.: A new formula of neutron multiplication during startup of PWR. Progr. Nucl. Energy 52, 321-326 (2010)

15. Sathiyasheela, T.: Sub-critical reactor kinetics analysis using incomplete gamma functions and binomial expansions. Ann. Nucl. Energy 37, 1248-1253 (2010)

16. Hetrick, D.L.: Dynamics of Nuclear Reactor, American Nuclear Society. Jbc, Illinois (1993)

17. Chen, W.Z., Kuang, B., Guoa, L.F., Chen, Z.Y., Zhu, B.: New analysis of prompt supercritical process with temperature feedback. Nucl. Eng. Design 236, 1326-1329 (2006)

18. Li, H., Chen, W.Z., Zhang, F., Luo, L.: Approximate solutions of point kinetics equations with one delayed neutron group and temperature feedback during delayed supercritical process. Ann. Nucl. Energy 34, 521-526 (2007)

19. Zhang, F., Chen, W.Z., Zhao, X.W.: The dynamic simulation of cold start-up based on two-group point reactor model. Ann. Nucl. Energy 36, 784-786 (2009)

20. Abramowitz, M., Stegun, I.A.: Handbook of mathmatical functions. National Bureau of Standards, Washington D.C. (1964)

21. Bhatt, T.U., Shimjith, S.R., Tiwari, A.P., Singh, K.P., Singh, S.K., Singh, K., Patil, R.K.: Estimation of sub-criticality using extended Kalman altering technique. Ann. Nucl. Energy 61, 98-115 (2013)

22. Jiang, S.Y., Yao, M.S., Bo, J.H., Wu, S.R.: Experimental simulation study on start-up of the $5 \mathrm{MW}$ nuclear heating reactor. Nucl. Eng. Design 158, 111-123 (1995) 